Poi nt wi se convergence of Cesar o and Ri esz means on certai $n$ funct $i$ on spaces

\begin{tabular}{|l|l|}
\hline 著者 & Sat o Shui chi \\
\hline $\begin{array}{l}\text { j our nal or } \\
\text { publ i cat i on t i t l e }\end{array}$ & Act a Sci ent i ar um Nat hemat i car um \\
\hline vol une & 80 \\
\hline number & $1-2$ \\
\hline page r ange & $129-139$ \\
\hline year & 2014-01- 01 \\
\hline URL & ht t p: //hdl . handl e. net /2297/43465 \\
\hline
\end{tabular}




\title{
Pointwise convergence of Cesàro and Riesz means on certain function spaces
}

\author{
Shuichi Sato
}

\section{Communicated by V. Totik}

\begin{abstract}
We consider a function space $\mathscr{Q} \mathscr{A}$ on the unit sphere of $\mathbb{R}^{3}$, which contains $L \log L \log \log \log L$, and prove the spherical harmonics expansions of functions in $\mathscr{Q} \mathscr{A}$ are summable a.e. with respect to the Cesàro means of the critical order $1 / 2$. We also prove that a similar result holds for the BochnerRiesz means of multiple Fourier series of periodic functions on $\mathbb{R}^{d}, d \geq 2$.
\end{abstract}

\section{Introduction}

Let

$$
Q_{d}=\left\{x \in \mathbb{R}^{d}:-1 / 2<x_{i} \leq 1 / 2, i=1,2, \ldots, d\right\}, \quad x=\left(x_{1}, \ldots, x_{d}\right),
$$

be the fundamental cube in the $d$-dimensional Euclidean space $\mathbb{R}^{d}$. For $f \in L^{1}\left(Q_{d}\right)$ we consider the Fourier series

$$
f(x) \sim \sum a_{n} e^{2 \pi i\langle n, x\rangle}, \quad n=\left(n_{1}, n_{2}, \ldots, n_{d}\right) \in \mathbb{Z}^{d},
$$

where $\langle n, x\rangle=n_{1} x_{1}+\cdots+n_{d} x_{d}$ and

$$
a_{n}=\int_{Q_{d}} f(x) e^{-2 \pi i\langle n, x\rangle} d x, \quad d x=d x_{1} \ldots d x_{d},
$$

is the Fourier coefficient. The Bochner-Riesz means of order $\delta$ of the series are defined by

$$
T_{R}^{\delta}(f)(x)=\sum_{|n|<R}\left(1-\frac{|n|^{2}}{R^{2}}\right)^{\delta} a_{n} e^{2 \pi i\langle n, x\rangle},
$$

Received May 21, 2012, and in revised form September 17, 2012.

AMS Subject Classifications: 42C10, 42B08.

Key words and phrases: spherical harmonics expansion, Cesàro means, Bochner-Riesz means. 
where $|n|=\left(n_{1}^{2}+\cdots+n_{d}^{2}\right)^{1 / 2}$.

According to [2], we define a space $\mathscr{Q} \mathscr{A}\left(Q_{d}\right)$ to be the collection of measurable functions $f$ for which we can find a sequence $\left\{f_{j}\right\}$ of non-negative measurable functions such that

$$
|f| \leq \sum_{j=1}^{\infty} f_{j}, \quad N\left(\left\{f_{j}\right\}\right):=\sum_{j=1}^{\infty}(1+\log j)\left\|f_{j}\right\|_{1} \log \left(\frac{e\left\|f_{j}\right\|_{\infty}}{\left\|f_{j}\right\|_{1}}\right)<\infty ;
$$

let $\|f\|_{\mathscr{Q} \mathscr{A}}=\inf N\left(\left\{f_{j}\right\}\right)$, where the infimum is taken over all such $\left\{f_{j}\right\}$. Then, the space $\mathscr{Q} \mathscr{A}$ is a $\log$ convex quasi-Banach space and a subspace of $L \log L$ (see $[2,9]$ ).

Define $T_{*}^{\delta}(f)(x)=\sup _{R>0}\left|T_{R}^{\delta}(f)(x)\right|$. Let $\alpha=(d-1) / 2$ (the critical index). Then we shall prove the following.

Theorem 1. There exists a positive constant $C$ such that

$$
\left\|T_{*}^{\alpha}(f)\right\|_{1, \infty}=\sup _{\lambda>0} \lambda\left|\left\{x \in Q_{d}: T_{*}^{\alpha}(f)(x)>\lambda\right\}\right| \leq C\|f\|_{\mathscr{Q} \mathscr{A}} ;
$$

consequently,

$$
\lim _{R \rightarrow \infty} T_{R}^{\alpha}(f)(x)=f(x) \quad \text { a.e. } \quad \text { for } f \in \mathscr{Q} \mathscr{A}\left(Q_{d}\right) .
$$

It is known that $L \log L \log \log \log L$ is a proper subspace of $\mathscr{Q} \mathscr{A}$ (see [2]). Thus, Theorem 1 implies the following.

Theorem 2. If $f \in L \log L \log \log \log L\left(Q_{d}\right)$, then

$$
\lim _{R \rightarrow \infty} T_{R}^{\alpha}(f)(x)=f(x) \quad \text { a.e. }
$$

The convergence a.e. for $f \in L \log L \log \log L\left(Q_{d}\right)$ was proved in [17].

If we write $T_{N}(f)=T_{N}^{\alpha}(f)$ when $d=1$, then $T_{N+1}(f)$ is the $N$ th partial sum of the Fourier series of $f$. For $f \in L^{2}\left(Q_{1}\right)$, there is a result of L. Carleson [5] which shows that $\left\{T_{N} f\right\}$ converges a.e. (see also [7]). Let $T_{*} f=\sup _{N \geq 1}\left|T_{N} f\right|$. R. Hunt [8] proved the restricted weak type estimates:

$$
\sup _{\lambda>0} \lambda\left|\left\{x \in Q_{1}: T_{*}\left(\chi_{A}\right)(x)>\lambda\right\}\right|^{1 / p} \leq C p^{2}(p-1)^{-1}|A|^{1 / p}, \quad 1<p<\infty,
$$

where $\chi_{A}$ denotes the characteristic function of a set $A \subset Q_{1}$. By (1.2) R. Hunt [8] proved the convergence a.e. of $\left\{T_{N} f\right\}$ for $f \in L(\log L)^{2}\left(Q_{1}\right)$. P. Sjölin [12] showed that (1.2) can be used to prove the convergence a.e. for the class $L \log L \log \log L\left(Q_{1}\right)$. Applying (1.2) more efficiently, N. Yu. Antonov [1] proved that $\left\{T_{N} f\right\}$ converges a.e. if $f \in L \log L \log \log \log L\left(Q_{1}\right)$. Theorem 2 can be regarded as a generalization of this result to higher dimensions.

To prove Theorem 1 for $d \geq 2$ we use the following estimates: 
Lemma 1. Let $1<p \leq 2, d \geq 2$. Then there exists a constant $C$ independent of $p$ such that

$$
\sup _{\lambda>0} \lambda\left|\left\{x \in Q_{d}: T_{*}^{\alpha}(f)(x)>\lambda\right\}\right|^{1 / p} \leq C(p-1)^{-1}\|f\|_{p} .
$$

We write $\delta=\sigma+i \tau, \sigma, \tau \in \mathbb{R}$. Lemma 1 was proved in [17] by using the following two results and analytic interpolation.

Lemma 2. Suppose $f \in L^{1}\left(Q_{d}\right), d \geq 2$ and $\sigma>\alpha$. Then

$$
\left\|T_{*}^{\delta}(f)\right\|_{1, \infty} \leq A_{\sigma} e^{\pi|\tau|}(\sigma-\alpha)^{-1}\|f\|_{1},
$$

where $A_{\sigma}$ remains bounded as $\sigma \rightarrow \alpha$.

Lemma 3. Suppose that $f \in L^{2}\left(Q_{d}\right), d \geq 2$. Then

$$
\left\|T_{*}^{\delta}(f)\right\|_{2} \leq A_{\sigma} e^{\pi|\tau|}\|f\|_{2}, \quad \sigma>0
$$

See Lemma 12 and Theorem 7 of [15] for Lemmas 2 and 3, respectively.

Sjölin-Soria [13] extended results of [1] to more general settings. We can apply results of [13] to prove Theorem 2 for $d \geq 2$. Indeed, we easily see that Theorem 2 for $d \geq 2$ follows from Lemma 1 and methods of [13, Section 3] (see Remark at the end of Section 3 of [13]). When $d=1$, Theorem 1 is due to [2]. The result also can be proved by using the estimate (1.2) and Antonov's idea. More precisely, when $d=1$, Lemma 7 (a key estimate) below is first proved for characteristic functions by applying (1.2) and the transition from characteristic functions to general functions $f$ can be carried out by Antonov's idea. We can prove Theorem 1 by Lemma 1 in the same way in higher dimensions. In fact, our proof of Theorem 1 for $d \geq 2$ is more straightforward; to prove Lemma 7 the application of the idea of Antonov is not needed, since the estimate of Lemma 1 is not restricted to characteristic functions (see Section 2).

We have analogous results for the Cesàro means of spherical harmonics expansions. Let $\mathscr{H}_{k}$ be the space of the spherical harmonics of degree $k$ on $\Sigma_{d}$, where $\Sigma_{d}=\left\{x \in \mathbb{R}^{d+1}:|x|=1\right\}$ is the unit sphere in $\mathbb{R}^{d+1}$. We recall that the space $\mathscr{H}_{k}$ consists of the restrictions to $\Sigma_{d}$ of harmonic homogeneous polynomials of degree $k$. Let

$$
H_{k} f(x)=\int_{\Sigma_{d}} Z_{x}^{(k)}(y) f(y) d \mu(y),
$$

where $d \mu$ is the Lebesgue surface measure on $\Sigma_{d}$ normalized as $\mu\left(\Sigma_{d}\right)=1$ (we also write $|E|=\mu(E)$ for a set $\left.E \subset \Sigma_{d}\right)$, and $Z_{x}^{(k)} \in \mathscr{H}_{k}$ is the zonal harmonic of degree 
$k$ with pole $x \in \Sigma_{d}$ :

$$
\begin{aligned}
Z_{x}^{(k)}(y) & =\left(\frac{2 k}{d-1}+1\right) \frac{\Gamma(d / 2) \Gamma(d+k-1)}{\Gamma(d-1) \Gamma(k+d / 2)} P_{k}^{((d-2) / 2,(d-2) / 2)}(\langle x, y\rangle) \\
& =\left(\frac{2 k}{d-1}+1\right) P_{k}^{((d-1) / 2)}(\langle x, y\rangle) .
\end{aligned}
$$

Here $P_{k}^{(\alpha, \beta)}$ is the Jacobi polynomial and $P_{k}^{(\lambda)}$ is the Gegenbauer polynomial defined by $\left(1-2 t r+r^{2}\right)^{-\lambda}=\sum_{k=0}^{\infty} P_{k}^{(\lambda)}(t) r^{k}$. We consider the spherical harmonics expansion $f \sim \sum_{k=0}^{\infty} H_{k} f$ and the Cesàro means of order $\delta$ defined by

$$
S_{n}^{\delta} f=\frac{1}{A_{n}^{(\delta)}} \sum_{k=0}^{n} A_{n-k}^{(\delta)} H_{k} f, \quad n=0,1,2, \ldots, \quad \delta=\sigma+i \tau,
$$

where

$$
A_{k}^{(\delta)}=\frac{\Gamma(k+\delta+1)}{\Gamma(k+1) \Gamma(\delta+1)}=\left(\begin{array}{c}
k+\delta \\
k
\end{array}\right), \quad \sigma>-1
$$

(see [19, Chap. III]). We refer to $[4,6,14,18]$ and [16, Chap. IV] for relevant results.

Let $S_{*}^{\delta}(f)(x)=\sup _{n>0}\left|S_{n}^{\delta}(f)(x)\right|$. If we define the space $\mathscr{Q} \mathscr{A}\left(\Sigma_{d}\right)$ analogously to $\mathscr{Q} \mathscr{A}\left(Q_{d}\right)$, we have the following result (we focus on the case $d=2$ ).

Theorem 3. There exists a positive constant $C$ such that

$$
\sup _{\lambda>0} \lambda\left|\left\{x \in \Sigma_{2}: S_{*}^{1 / 2}(f)(x)>\lambda\right\}\right| \leq C\|f\|_{\mathscr{Q} \mathscr{A}}
$$

for $f \in \mathscr{Q} \mathscr{A}\left(\Sigma_{2}\right)$, which implies

$$
\lim _{n \rightarrow \infty} S_{n}^{1 / 2}(f)(x)=f(x) \quad \text { a.e. } \quad \text { for } f \in \mathscr{Q} \mathscr{A}\left(\Sigma_{2}\right) .
$$

Theorem 3 implies the following result as Theorem 1 implies Theorem 2.

Theorem 4. If $f \in L \log L \log \log \log L\left(\Sigma_{2}\right)$, then

$$
\lim _{n \rightarrow \infty} S_{n}^{1 / 2} f(x)=f(x) \quad \text { a.e. }
$$

See [4] for the convergence a.e. of $\left\{S_{n}^{1 / 2} f\right\}$ for $f \in L^{p}\left(\Sigma_{2}\right), p>1$. The proof of Theorem 3 is similar to that of Theorem 1, with the following estimates:

Lemma 4. Let $1<p \leq 2$. Then we have

$$
\sup _{\lambda>0} \lambda\left|\left\{x \in \Sigma_{2}: S_{*}^{1 / 2}(f)(x)>\lambda\right\}\right|^{1 / p} \leq C(p-1)^{-1}\|f\|_{p}
$$

for a positive constant $C$ independent of $p$. 
Let

$$
M f(x)=\sup _{r>0}|B(x, r)|^{-1} \int_{B(x, r)}|f(y)| d \mu(y),
$$

where $B(x, r)=\left\{y \in \Sigma_{2}:|y-x|<r\right\}, x \in \Sigma_{2}$. To prove Lemma 4 we need the following two results.

Lemma 5. Suppose that $f \in L^{1}\left(\Sigma_{2}\right)$ and $\alpha<\sigma<1$, where $\alpha=1 / 2$. Then

$$
S_{*}^{\delta}(f)(x) \leq A_{\sigma} e^{B \tau^{2}}(\sigma-\alpha)^{-1}(M f(x)+M f(-x)) .
$$

The constant $A_{\sigma}$ remains bounded as $\sigma \rightarrow \alpha$.

Lemma 6. Suppose that $f \in L^{2}\left(\Sigma_{2}\right)$. Then

$$
\left\|S_{*}^{\delta}(f)\right\|_{2} \leq A_{\sigma} e^{B_{\sigma} \tau^{2}}\|f\|_{2}, \quad \sigma>0 .
$$

The constants $A_{\sigma}$ and $B_{\sigma}$ are bounded on any compact subinterval of $(0, \infty)$.

We can find Lemma 6 in [4]. Using Lemmas 5 and 6, we can prove Lemma 4 by analytic interpolation (see Section 4). We shall prove Lemma 5 in Section 3 by applying methods of [10].

\section{Proof of Theorem 1}

We assume that $d \geq 2$. In proving Theorem 1 we use the following result.

Lemma 7. Suppose that $f \in L^{\infty}\left(Q_{d}\right), f \neq 0$. Then

$$
\left\|T_{*}^{\alpha}(f)\right\|_{1, \infty} \leq C\|f\|_{1} \log \left(\frac{e\|f\|_{\infty}}{\|f\|_{1}}\right)
$$

Proof. By homogeneity we may assume that $\|f\|_{\infty}=1$. For $\lambda>0$, let $m(\lambda)=$ $\inf _{1<p \leq 2} \lambda^{-p}(p-1)^{-p}$. Then, observing that $\|f\|_{p}^{p} \leq\|f\|_{1}$, by Lemma 1 we have

$$
\left|\left\{x \in Q_{d}: T_{*}^{\alpha}(f)(x)>\lambda\right\}\right| \leq C \min \left(1, m(\lambda)\|f\|_{1}\right) .
$$

This will imply the conclusion, if we note that $m(\lambda)=\lambda^{-2}$ when $\lambda \geq e^{-2}$ and $m(\lambda) \sim \lambda^{-1} \log (1 / \lambda)$ when $\lambda<e^{-2}$.

Let $f \in \mathscr{Q} \mathscr{A}\left(Q_{d}\right)$. To prove Theorem 1 , we may assume that $f \geq 0$. For any $\epsilon>0$ there exists a sequence $\left\{f_{j}\right\}$ of non-negative bounded functions such that $f=\sum f_{j}$ and $N\left(\left\{f_{j}\right\}\right) \leq\|f\|_{\mathscr{Q} \mathscr{A}}+\epsilon$ (see [2, p. 149]). Since $L^{1, \infty}$ is a logconvex 
quasi-Banach space (see [9]) and $T_{*}^{\alpha}$ is a sublinear operator, using Lemma 7 we have

$$
\begin{aligned}
\left\|T_{*}^{\alpha}(f)\right\|_{1, \infty} & \leq C \sum_{j}(1+\log j)\left\|T_{*}^{\alpha}\left(f_{j}\right)\right\|_{1, \infty} \\
& \leq C \sum_{j}(1+\log j)\left\|f_{j}\right\|_{1} \log \left(\frac{e\left\|f_{j}\right\|_{\infty}}{\left\|f_{j}\right\|_{1}}\right)=C N\left(\left\{f_{j}\right\}\right) \leq C\left(\|f\|_{\mathscr{Q} \mathscr{A}}+\epsilon\right) .
\end{aligned}
$$

Letting $\epsilon \rightarrow 0$, we get the conclusion.

\section{Proof of Lemma 5}

Let

$$
S_{n}^{(\delta, \lambda)}(\cos v)=\left(A_{n}^{(\delta)}\right)^{-1} \sum_{k=0}^{n} A_{n-k}^{(\delta)} 2(k+\lambda) P_{k}^{(\lambda)}(\cos v)
$$

where $0<\lambda<1,0 \leq v \leq \pi, 0<\sigma<1, \delta=\sigma+i \tau$. Then, $S_{n}^{(\delta, 1 / 2)}(\langle x, y\rangle)$ is the kernel of the operator $S_{n}^{\delta}$. In [10, p. 121], $S_{n}^{(\delta, \lambda)}(\cos v)$ was represented by the contour integrals as follows:

$$
\frac{1}{2} A_{n}^{(\delta)} S_{n}^{(\delta, \lambda)}(\cos v)=\frac{1}{2 \pi i} \int_{L_{1}} \varphi(z) d z+\frac{1}{2 \pi i} \int_{L_{2}} \varphi(z) d z+\frac{1}{2 \pi i} \int_{L_{3}} \varphi(z) d z
$$

where

$$
\varphi(z)=\frac{\lambda(1+z) z^{n+\delta+2 \lambda}}{(z-1)^{\delta}\left(1-2 z \cos v+z^{2}\right)^{\lambda+1}} .
$$

Let

$$
\begin{aligned}
i_{n}^{(\delta, \lambda)}(v)= & \frac{\lambda \sin (\delta \pi)}{\pi} \int_{0}^{1} \frac{u^{n+\delta+2 \lambda}}{(1-u)^{\delta}\left(1-2 u \cos v+u^{2}\right)^{\lambda+1}} d u \\
\mathscr{I}_{n}^{(\delta, \lambda)}(v)= & \frac{\exp (-i[(n+\lambda+(\delta+1) / 2) v-(\lambda+\delta+1) \pi / 2])}{(2 \sin v)^{\lambda}(2 \sin (v / 2))^{\delta+1}} \frac{\sin (\lambda \pi)}{\pi} \times \\
& \times \int_{0}^{1} \frac{u^{-\lambda}(1-u)^{n+\delta+2 \lambda}}{(1-u \tau(v / 2))^{\delta+1}(1-u \tau(v))^{\lambda}} d u \\
\mathscr{J}_{n}^{(\delta, \lambda)}(v)= & \frac{\exp (i[(n+\lambda+(\delta+1) / 2) v-(\lambda+\delta+1) \pi / 2])}{\sin (\lambda \pi)} \frac{(2 \sin v)^{\lambda}(2 \sin (v / 2))^{\delta+1}}{\pi} \times \\
& \times \int_{0}^{1} \frac{u^{-\lambda}(1-u)^{n+\delta+2 \lambda}}{(1-u \tau(-v / 2))^{\delta+1}(1-u \tau(-v))^{\lambda}} d u
\end{aligned}
$$


where $\tau(v)=(1+i \cot v) / 2$. Then, according to (3.1), it follows that

$$
\begin{aligned}
\frac{1}{2} A_{n}^{(\delta)} S_{n}^{(\delta, \lambda)}(\cos v)=( & +\lambda) \mathscr{I}_{n}^{(\delta, \lambda)}(v)-(\delta+1) \mathscr{I}_{n-1}^{(\delta+1, \lambda)}(v)+i_{n+1}^{(\delta, \lambda)}(v)+ \\
& +i_{n}^{(\delta, \lambda)}(v)+(n+\lambda) \mathscr{J}_{n}^{(\delta, \lambda)}(v)-(\delta+1) \mathscr{J}_{n-1}^{(\delta+1, \lambda)}(v)
\end{aligned}
$$

(see [10]). Put

$$
\begin{aligned}
K(n, \delta, \lambda, v) & =\frac{4(n+\lambda)}{\Gamma(\lambda)} C(n, \delta, \lambda) \frac{\cos [(n+\lambda+(\delta+1) / 2) v-(\lambda+\delta+1) \pi / 2]}{(2 \sin v)^{\lambda}(2 \sin (v / 2))^{\delta+1}}, \\
L(n, \delta, \lambda, v) & =\frac{-4(\delta+1)}{\Gamma(\lambda)} C(n, \delta, \lambda) \frac{\cos [(n+\lambda+\delta / 2) v-(\lambda+\delta+2) \pi / 2]}{(2 \sin v)^{\lambda}(2 \sin (v / 2))^{\delta+2}},
\end{aligned}
$$

where

$$
C(n, \delta, \lambda)=\frac{\Gamma(n+\delta+2 \lambda+1)}{\Gamma(n+\delta+\lambda+2)}
$$

and also

$$
\begin{aligned}
& R_{1}(n, \delta, \lambda, v)=2(n+\lambda) \mathscr{I}_{n}^{(\delta, \lambda)}(v)+2(n+\lambda) \mathscr{J}_{n}^{(\delta, \lambda)}(v)-K(n, \delta, \lambda, v), \\
& R_{2}(n, \delta, \lambda, v)=-2(\delta+1) \mathscr{I}_{n-1}^{(\delta+1, \lambda)}(v)-2(\delta+1) \mathscr{J}_{n-1}^{(\delta+1, \lambda)}(v)-L(n, \delta, \lambda, v), \\
& R_{3}(n, \delta, \lambda, v)=2 i_{n+1}^{(\delta, \lambda)}(v)+2 i_{n}^{(\delta, \lambda)}(v) .
\end{aligned}
$$

Then (3.2) implies that

$$
\begin{aligned}
S_{n}^{(\delta, \lambda)}(\cos v)=\left(A_{n}^{(\delta)}\right)^{-1}(K(n, \delta, \lambda, v) & +L(n, \delta, \lambda, v)+R_{1}(n, \delta, \lambda, v)+ \\
& \left.+R_{2}(n, \delta, \lambda, v)+R_{3}(n, \delta, \lambda, v)\right) .
\end{aligned}
$$

We need the following results.

Lemma 8. Let $x>-1, y \in \mathbb{R}$. Then $\left|A_{n}^{(x+i y)}\right| \geq\left|A_{n}^{(x)}\right|$ and $\left|A_{n}^{(x+i y)}\right| \leq e^{c(x) y^{2}} A_{n}^{(x)}$, where $c(x)=(1 / 2) \sum_{k=1}^{\infty}(x+k)^{-2}$ and $A_{n}^{(x+i y)}$ is as in (1.3).

Lemma 9. Suppose $0<\lambda<1,0<\sigma<1$. Let $C(n, \delta, \lambda)$ be as above. Then

$$
|C(n, \delta, \lambda)| \leq C(n+1)^{\lambda-1}
$$

where the constant $C$ is independent of $\delta$ and $\lambda$.

Lemma 8 is in [3]. Lemma 9 can be proved by using the formula

$$
\lim _{\operatorname{Re}(z) \geq c>0,|z| \rightarrow \infty} \frac{\Gamma(z)}{\sqrt{2 \pi} e^{-z} z^{z-1 / 2}}=1 .
$$


Let $|\pi / 2-v| \leq(\pi / 2)(n /(n+1))$. By [10, pp. 130-133] and Lemma 9 we have

$$
\begin{aligned}
\left|R_{1}(n, \delta, \lambda, v)\right| & \leq C e^{B|\tau|} \frac{C(n, \sigma, \lambda)}{\Gamma(\lambda)|n+\sigma+\lambda+2|} \frac{n+1}{(\sin v)^{\lambda+1}(\sin (v / 2))^{\sigma+1}} \\
& \leq C e^{B|\tau|} \frac{(n+1)^{\lambda-1}}{(\sin v)^{\lambda+1}(\sin (v / 2))^{\sigma+1}}, \\
\left|R_{2}(n, \delta, \lambda, v)\right| & \leq C e^{B|\tau|} \frac{C(n, \sigma, \lambda)}{\Gamma(\lambda)|n+\sigma+\lambda+2|} \frac{1}{(\sin v)^{\lambda+1}(\sin (v / 2))^{\sigma+2}} \\
& \leq C e^{B|\tau|} \frac{(n+1)^{\lambda-1}}{(\sin v)^{\lambda+1}(\sin (v / 2))^{\sigma+1}} .
\end{aligned}
$$

Also, by [10, pp. 122-123] and estimates similar to the one in Lemma 9

$$
\begin{aligned}
\left|R_{3}(n, \delta, \lambda, v)\right| & \leq C \frac{|\sin (\delta \pi)| \Gamma(1-\sigma)}{(\sin (v / 2))^{2(\lambda+1)}}\left(\frac{\Gamma(n+\sigma+2 \lambda+1)}{\Gamma(n+2 \lambda+2)}+\frac{\Gamma(n+\sigma+2 \lambda+2)}{\Gamma(n+2 \lambda+3)}\right) \\
& \leq C(n+1)^{\sigma-1} \frac{|\sin (\delta \pi)| \Gamma(1-\sigma)}{(\sin (v / 2))^{2(\lambda+1)}} .
\end{aligned}
$$

Since $\left|A_{n}^{(\delta)}\right| \geq\left|A_{n}^{(\sigma)}\right|$ and $A_{n}^{(\sigma)} \sim(n+1)^{\sigma}$ (see Lemma 8 and [19, Chap. III]), if $|\pi / 2-v| \leq(\pi / 2)(n /(n+1))$, we have

$$
\begin{aligned}
& \left|R_{j}(n, \delta, \lambda, v) / A_{n}^{(\delta)}\right| \leq C e^{B|\tau|} \frac{(n+1)^{\lambda-1-\sigma}}{(\sin v)^{\lambda+1}(\sin (v / 2))^{\sigma+1}}, \quad j=1,2, \\
& \left|R_{3}(n, \delta, \lambda, v) / A_{n}^{(\delta)}\right| \leq C \frac{|\sin (\delta \pi)| \Gamma(1-\sigma)}{(n+1)(\sin (v / 2))^{2(\lambda+1)}}
\end{aligned}
$$

By Lemma 9 we have

$$
\left|K(n, \delta, \lambda, v) / A_{n}^{(\delta)}\right| \leq C e^{(\pi / 2)|\tau|} \frac{(n+1)^{\lambda-\sigma}}{(\sin v)^{\lambda}(\sin (v / 2))^{\sigma+1}} .
$$

Similarly,

$$
\left|L(n, \delta, \lambda, v) / A_{n}^{(\delta)}\right| \leq C(1+|\tau|) e^{(\pi / 2)|\tau|} \frac{(n+1)^{\lambda-\sigma-1}}{(\sin v)^{\lambda}(\sin (v / 2))^{\sigma+2}}
$$

We also need the following.

Lemma 10. Let $0<\lambda<1,0<\sigma<1, \delta=\sigma+i \tau, 0 \leq v \leq \pi$. Then

$$
\left|S_{n}^{(\delta, \lambda)}(\cos v)\right| \leq C e^{c \tau^{2}}(n+1)^{2 \lambda+1}
$$


Proof. By [18, p. 168], we have $\left|P_{n}^{(\lambda)}\right| \leq C A_{n}^{(2 \lambda-1)}$. Using this and Lemma 8, we see that

$$
\begin{aligned}
\left|S_{n}^{(\delta, \lambda)}(\cos v)\right| & \leq C\left|A_{n}^{(\delta)}\right|^{-1} \sum_{m=0}^{n}\left|A_{n-m}^{(\delta)}\right|(m+\lambda) A_{m}^{(2 \lambda-1)} \\
& \leq C \lambda\left|A_{n}^{(\delta)}\right|^{-1} \sum_{m=0}^{n} \frac{m+\lambda}{m+2 \lambda}\left|A_{n-m}^{(\delta)}\right| A_{m}^{(2 \lambda)} \\
& \leq C e^{c \tau^{2}}\left|A_{n}^{(\sigma)}\right|^{-1} \sum_{m=0}^{n}\left|A_{n-m}^{(\sigma)}\right| A_{m}^{(2 \lambda)} \\
& \leq C e^{c \tau^{2}}\left|A_{n}^{(\sigma)}\right|^{-1} A_{n}^{(\sigma+2 \lambda+1)} \leq C e^{c \tau^{2}}(n+1)^{2 \lambda+1} .
\end{aligned}
$$

By (3.3)-(3.7) and Lemma 10, we have

$$
\left|S_{n}^{(\delta, \lambda)}(\cos v)\right| \leq C e^{B \tau^{2}}(n+1)^{\lambda-\sigma}\left((n+1)^{-1}+\sin v\right)^{-\lambda-\sigma-1},
$$

where $0 \leq v \leq \pi, \lambda=1 / 2,1 / 2<\sigma<1$. Suppose $\langle x, y\rangle=\cos v, x, y \in \Sigma_{2}$. Then $\sin v \sim|x-y|$ if $\langle x, y\rangle \geq 0$ and $\sin v \sim|x+y|$ if $\langle x, y\rangle \leq 0$. Thus (3.8) implies

$$
\begin{aligned}
& \left|S_{n}^{(\delta, \lambda)}(\langle x, y\rangle)\right| \\
& \quad \leq \begin{cases}C e^{B \tau^{2}}(n+1)^{\lambda-\sigma}\left((n+1)^{-1}+|x-y|\right)^{-\lambda-\sigma-1}, & \text { if }\langle x, y\rangle \geq 0, \\
C e^{B \tau^{2}}(n+1)^{\lambda-\sigma}\left((n+1)^{-1}+|x+y|\right)^{-\lambda-\sigma-1}, & \text { if }\langle x, y\rangle \leq 0 .\end{cases}
\end{aligned}
$$

Since $S_{n}^{\delta} f(x)=\int_{\Sigma_{2}} S_{n}^{(\delta, 1 / 2)}(\langle x, y\rangle) f(y) d \mu(y)$, the conclusion of Lemma 5 easily follows from (3.9).

Remark. In fact, we can prove estimates of the type in [6, Theorem (3.21)], partly improving (3.9). We do not need those estimates here; for our purpose (3.9) suffices.

\section{Proofs of Lemmas 4, 6 and Theorem 3}

We first prove Lemma 6 .

Proof of Lemma 6. When $\delta>0$, we have $\left\|S_{*}^{\delta}(f)\right\|_{2} \leq A_{\delta}\|f\|_{2}$ (see [4, Lemma $(3.5)])$. If $\delta=\sigma+i \tau, \sigma>0, \tau \in \mathbb{R}$, we write

$$
S_{n}^{\delta}(f)=\left(A_{n}^{\delta}\right)^{-1} \sum_{k=0}^{n} A_{k}^{(\sigma-\epsilon)} A_{n-k}^{(\epsilon-1+i \tau)} S_{k}^{\sigma-\epsilon}(f),
$$

where $0<\epsilon<\sigma$. Using Lemma 8 as in [4], we have $S_{*}^{\delta}(f) \leq e^{c(\epsilon-1) \tau^{2}} S_{*}^{\sigma-\epsilon}(f)$. Combining these results, we reach the conclusion of Lemma 6. 
Proof of Lemma 4. Let $1<p<2,1 / p=(1-\theta) / 2+\theta, \alpha=(1-\theta) c+\theta b$, where $c=\alpha-(1 / 2)(1 / p-1 / 2), b=\alpha+(1 / 2)(1-1 / p), \alpha=1 / 2$. We note that $\theta=2(1 / p-1 / 2), 1 / 4 \leq c \leq \alpha, \alpha \leq b \leq 3 / 4$.

Define $T_{z} f=S_{0}^{\delta(z)} f, \delta(z)=(1-z) c+z b, 0 \leq \sigma \leq 1, z=\sigma+i \tau, \tau \in \mathbb{R}$. Here $S_{0}^{\delta}$ is a linear operator approximating $S_{*}^{\delta}$ defined by $S_{0}^{\delta} f(x)=S_{n(x)}^{\delta} f(x)$, where $n(x)$ is a suitable non-negative mapping from $\Sigma_{2}$ to $\mathbb{Z}$, so that $\left\{T_{z}\right\}$ is an analytic family of linear operators which is admissible in the sense of [11] (see also [16, Chap. V, Section 4]).

We apply the analytic interpolation theorem on the Lorentz spaces $L^{p, q}$ due to [11]. Note that $\operatorname{Re}(\delta(i \tau))=c \in[1 / 4,1 / 2]$. Thus Lemma 6 implies

$$
\left\|T_{i \tau} f\right\|_{2,2} \leq C_{0} e^{B_{0} \tau^{2}}\|f\|_{2,2}
$$

for some $B_{0}, C_{0}>0$. By Lemma 5 and the $L^{1}-L^{1, \infty}$ boundedness of the maximal operator $M$ we have

$$
\left\|T_{1+i \tau} f\right\|_{1, \infty} \leq C_{1}(p-1)^{-1} e^{B_{1} \tau^{2}}\|f\|_{1,1}
$$

for some $B_{1}, C_{1}>0$, since $\operatorname{Re}(\delta(1+i \tau))=b$. Interpolating between (4.1) and (4.2), we get

$$
\left\|S_{0}^{\alpha} f\right\|_{p, p^{\prime}}=\left\|T_{\theta} f\right\|_{p, p^{\prime}} \leq A_{\theta}\|f\|_{p, p}
$$

where

$$
A_{\theta} \leq C(p-1)^{-\theta} \leq C(p-1)^{-1}
$$

Therefore

$$
\left\|S_{0}^{\alpha} f\right\|_{p, \infty} \leq C\left\|S_{0}^{\alpha} f\right\|_{p, p^{\prime}} \leq C(p-1)^{-1}\|f\|_{p},
$$

from which Lemma 4 follows.

To prove Theorem 3, we note that by Lemma 4, similarly to the case of $T_{*}^{\alpha}$, we can prove

$$
\left\|S_{*}^{1 / 2} f\right\|_{1, \infty} \leq C\|f\|_{1} \log \left(\frac{e\|f\|_{\infty}}{\|f\|_{1}}\right)
$$

if $f \in L^{\infty}\left(\Sigma_{2}\right), f \neq 0$. Also, as in the case of $T_{*}^{\alpha}$, the estimate (4.3) readily implies $\left\|S_{*}^{1 / 2} f\right\|_{1, \infty} \leq C\|f\|_{\mathscr{Q} A}$, from which the almost everywhere convergence follows.

\section{References}

[1] N. Yu. Antonov, Convergence of Fourier series, East J. Approx., 2 (1996), 187-196.

[2] J. Arias-de-Reyna, Pointwise convergence of Fourier series, J. London Math Soc. (2), 65 (2002), 139-153. 
[3] R. Askey and I. I. Hirschman, Mean summability for ultraspherical polynomials, Math. Scand., 12 (1963), 167-177.

[4] A. Bonami and J.-L. Clerc, Sommes de Cesàro et multiplicateurs des développments en harmonique sphérique, Trans. Amer. Math. Soc., 183 (1973), 223-263.

[5] L. Carleson, On convergence and growth of partial sums of Fourier series, Acta Math., 116 (1966), 135-157.

[6] L. Colzani, M. H. Taibleson and G. Weiss, Maximal estimates for Cesàro and Riesz means on spheres, Indiana Univ. Math. J., 33 (1984), 873-889.

[7] C. Fefferman, Pointwise convergence of Fourier series, Ann. of Math., 98 (1973), $551-572$.

[8] R. Hunt, On the convergence of Fourier series, Orthogonal Expansions and their Continuous Analogues (Edwardsville, IL, 1967), Southern Illinois Univ. Press, 1968, 235-255.

[9] N. J. Kalton, Convexity type and the three space problem, Studia Math., 69 (1981), 247-287.

[10] E. Kogbetliantz, Recherches sur la sommabilité des séries ultrasphériques par la méthode des moyennes arithmétiques, J. Math. Pures Appl., 3 (1924), 107-187.

[11] Y. Sagher, On analytic families of operators, Israel J. Math., 7 (1969), 350-356.

[12] P. SJöLIN, An inequality of Paley and convergence a.e. of Walsh-Fourier series, Ark. Mat., 7 (1968), 551-570.

[13] P. Suölin and F. Soria, Remarks on a theorem by N. Yu. Antonov, Studia Math., 158 (2003), 79-97.

[14] C. D. Sogge, Oscillatory integrals and spherical harmonics, Duke Math. J., 53 (1986), 43-65.

[15] E. M. Stein, Localization and summability of multiple Fourier series, Acta Math., 100 (1958), 93-147.

[16] E. M. Stein and G. Weiss, Fourier Analysis on Euclidean Spaces, Princeton Univ. Press, Princeton, 1971.

[17] G. Sunouchi, On the summability almost everywhere of the multiple Fourier series at the critical index, Kodai Math. J., 8 (1985), 1-4.

[18] G. Szegö, Orthogonal Polynomials, 4th ed., Amer Math. Soc. Coll. Publ. No. 23, Amer. Math. Soc., Providence, R. I., 1975.

[19] A. Zygmund, Trigonometric Series, 2nd ed., Cambridge Univ. Press, CambridgeLondon - New York - Melbourne, 1977.

S. SAto, Department of Mathematics, Faculty of Education, Kanazawa University, Kanazawa 920-1192, Japan; e-mail: shuichi@kenroku.kanazawa-u.ac.jp 